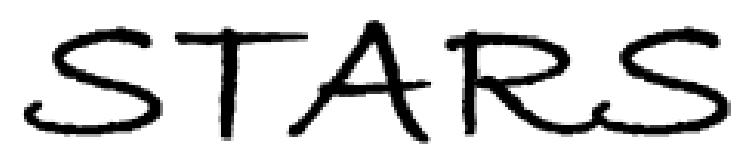

University of Central Florida

STARS

Faculty Bibliography 2000s

Faculty Bibliography

$1-1-2003$

\title{
Normal-mode anisotropic liquid-crystal gels
}

\author{
Yun-Hsing Fan \\ University of Central Florida \\ Hongwen Ren \\ University of Central Florida \\ Shin-Tson Wu \\ University of Central Florida
}

Find similar works at: https://stars.library.ucf.edu/facultybib2000 University of Central Florida Libraries http://library.ucf.edu

This Article is brought to you for free and open access by the Faculty Bibliography at STARS. It has been accepted for inclusion in Faculty Bibliography 2000 s by an authorized administrator of STARS. For more information, please contactSTARS@ucf.edu.

\section{Recommended Citation}

Fan, Yun-Hsing; Ren, Hongwen; and Wu, Shin-Tson, "Normal-mode anisotropic liquid-crystal gels" (2003). Faculty Bibliography 2000s. 3741.

https://stars.library.ucf.edu/facultybib2000/3741

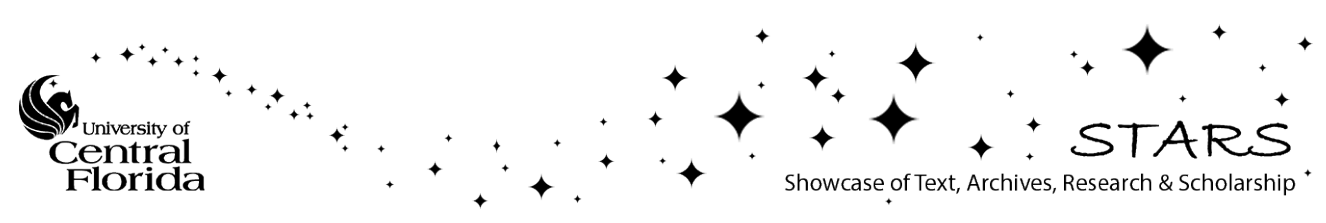




\section{Normal-mode anisotropic liquid-crystal gels}

Cite as: Appl. Phys. Lett. 82, 2945 (2003); https://doi.org/10.1063/1.1571663

Submitted: 20 December 2002 . Accepted: 07 March 2003 . Published Online: 29 April 2003

Yun-Hsing Fan, Hongwen Ren, and Shin-Tson Wu

\section{ARTICLES YOU MAY BE INTERESTED IN}

Anisotropic liquid crystal gels for switchable polarizers and displays

Applied Physics Letters 81, 1432 (2002); https://doi.org/10.1063/1.1502021

Dual-frequency liquid crystal gels with submillisecond response time

Applied Physics Letters 85, 2451 (2004); https://doi.org/10.1063/1.1799249

Cholesteric liquid crystal/polymer dispersion for haze-free light shutters

Applied Physics Letters 60, 3102 (1992); https://doi.org/10.1063/1.106765

\section{Applied Physics Letters}

Mid-IR and THz frequency combs special collection 


\title{
Normal-mode anisotropic liquid-crystal gels
}

\author{
Yun-Hsing Fan, Hongwen Ren, and Shin-Tson $\mathrm{Wu}^{\mathrm{a})}$ \\ School of Optics/CREOL, University of Central Florida, Orlando, Florida 32816
}

(Received 20 December 2002; accepted 7 March 2003)

\begin{abstract}
A normal-mode anisotropic liquid-crystal (LC) gel is demonstrated. In the absence of an electric field, the light polarized along the rubbing direction is scattered. In the voltage-on state, the gel is highly transparent and is independent of polarization. Such a normal mode LC gel exhibits a modest contrast ratio, response time, and low operating voltage. It can be used as a broadband switchable polarizer, variable optical attenuator, and reflective display. (c) 2003 American Institute of Physics.
\end{abstract}

[DOI: $10.1063 / 1.1571663$ ]

Liquid-crystal (LC) gel ${ }^{1,2}$ exhibits an anisotropic light scattering which is different from the polymer dispersed LC. To form an LC gel, a few percent $(3 \%-8 \%)$ of diacrylate monomer is mixed in a nematic LC host. The mixture is injected into an empty LC cell with surface rubbing and then exposed to a weak ultraviolet light. For a reversed-mode LC gel,,$^{3}$ the gel is highly transparent in the absence of an electric field. In the voltage-on state, the polymer networks and the electric field are working against each other resulting in multidomain structures that scatter light with polarization parallel to the rubbing direction. The polarization which is perpendicular to the rubbing direction is transmitted. Such an LC gel can be used for transflective display, broadband switchable polarizer, and variable optical attenuators for telecommunication. ${ }^{4}$

In this letter, we demonstrate method for preparing a normal-mode anisotropic LC gel and explore its electro-optic properties. The term "normal mode" refers to that the gel is in the scattering state at $V=0$ and is highly transparent in a high-voltage state. For display applications, the normalmode LC gel leads to a normally black display.

In our studies, the LC gel was fabricated by photopolymerizing a nematic LC host (Merck E48, $\Delta n=0.231$ ) and photocurable monomer. In the reverse mode, the monomer concentration $(c)$ is typically in the $3-10 \mathrm{wt} \%$ range. However, to obtain a normal mode, the monomer concentration is below $1 \%$ and an electric field needs to be applied during polymerization process. Four samples with $c=0.4,0.6,0.8$, and $1.0 \mathrm{wt} \%$ were prepared. The monomer used in this study is bisphenol-A-dimethacrylate with structure shown as follows:

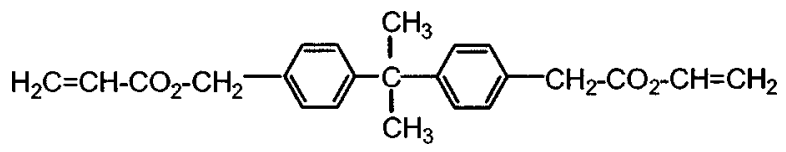

The LC/monomer mixture was filled in the cells with gap $d=10 \mu \mathrm{m}$. The inner surfaces of the indium-tin-oxide glass substrates were overcoated with a thin polyimide layer and buffed in antiparallel directions for achieving homogeneous alignment. The cell was exposed to UV light under $10 \mathrm{~V}$ biased voltage such that the LC directors were oriented to the electric-field direction and the monomers were polymerized

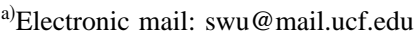

along the same direction which is perpendicular to the substrates. To avoid incomplete polymerization, a weak UV intensity $\left(I \sim 30 \mu \mathrm{W} / \mathrm{cm}^{2}\right)$ was used. The exposure time was 3 $\mathrm{h}$. The resulting polymer network was perpendicular to the substrate surface. Weak UV exposure and a slow polymerization process were found to be crucial to achieve a better dark state, higher contrast ratio, and lower operating voltage. Slow polymerization coarsened the polymer network, stabilized the polymer network, and enlarged the LC domains. ${ }^{5}$

The electro-optic properties of the LC gels were studied by measuring the transmittance of the LC gels with a $\mathrm{He}-\mathrm{Ne}$ laser. The laser beam diameter is about $1 \mathrm{~mm}$. A photodiode detector was set at $\sim 30 \mathrm{~cm}$ away from the LC sample. A sheet polarizer with its optical axis parallel to the rubbing direction of the substrate was put in front of the LC cell. The voltage-dependent transmittance of the $\mathrm{LC}$ gels is shown in Fig. 1 for $c=0.4,0.6,0.8$, and $1.0 \mathrm{wt} \%$ and $d=10 \mu \mathrm{m}$.

Figure 1 shows the voltage-dependent normalized transmittance of the $\mathrm{He}-\mathrm{Ne}$ laser beam polarized in the rubbing direction. The absolute transmittance of the on state reaches $98 \%$ if the multiple reflections of the substrates are excluded. Figure 2 shows the schematic diagrams illustrating the molecular arrangements of the voltage-off and -on states of the normal-mode LC gel. At $V=0$, the buffed polyimide layers tend to align $\mathrm{LC}$ molecules parallel to the rubbing direction, but the polymer networks tend to retain the LC directors perpendicular to the substrate. As a result, multiple domains are formed. The light polarized along the rubbing direction is

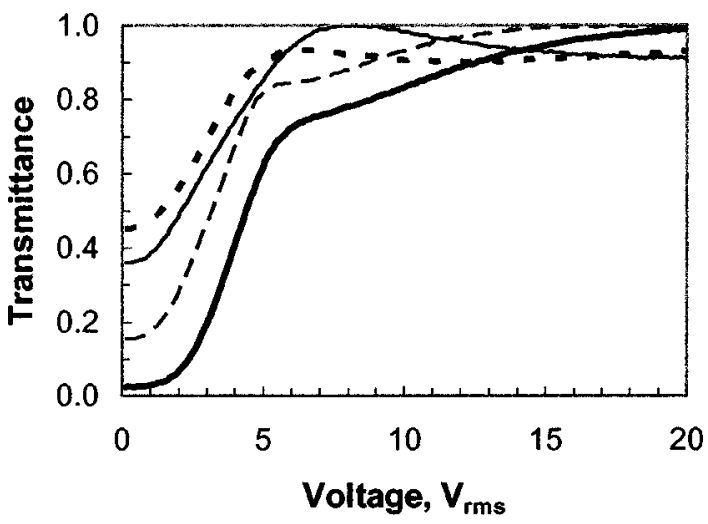

FIG. 1. Voltage-dependent normalized transmittance of the LC gels at different monomer concentrations in a $10 \mu \mathrm{m}$ cell. From top to bottom, $c$ $=1.0,0.8,0.6$, and $0.4 \mathrm{wt} \%$. 


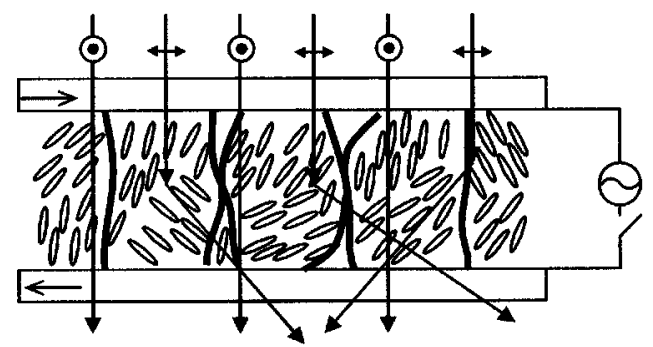

(a) Voltage off

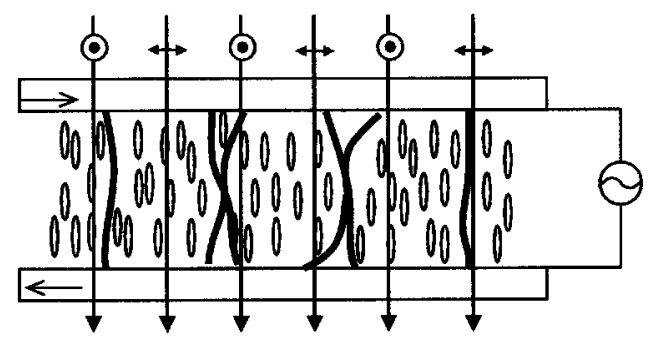

(b) Voltage on

FIG. 2. Schematic representation of the operating principle of LC gels: (a) voltage-off state and (b) voltage-on state. The arrows in the substrates indicate the rubbing directions.

scattered while the perpendicular component is transmitted without scattering.

From Fig. 1, monomer concentration plays an important role in determining the dark state of the normal-mode LC gel. The lower monomer concentration leads to a better dark state. For a higher monomer concentration, the anchoring force of the polymer network is stronger so that more LC molecules are aligned perpendicular to the substrates. Under such a circumstance, the gel is more ordered and its light scattering capability is weaker. As the monomer concentration is reduced to a certain level, the network anchoring force is comparable to the polyimide-induced alignment force. As a result, the gel is highly disordered and light scattering dominates in the voltage-off state. From Fig. 1, the measured contrast ratio is $\sim 100: 1$ for the sample with $0.4 \%$ monomer concentration. If we stack two anisotropic gels in orthogonal directions, the stacked cells transmit the input unpolarized light also with high efficiency. When one of the cells is activated, the output light becomes linearly polarized. When both cells are activated, both polarizations are scattered and

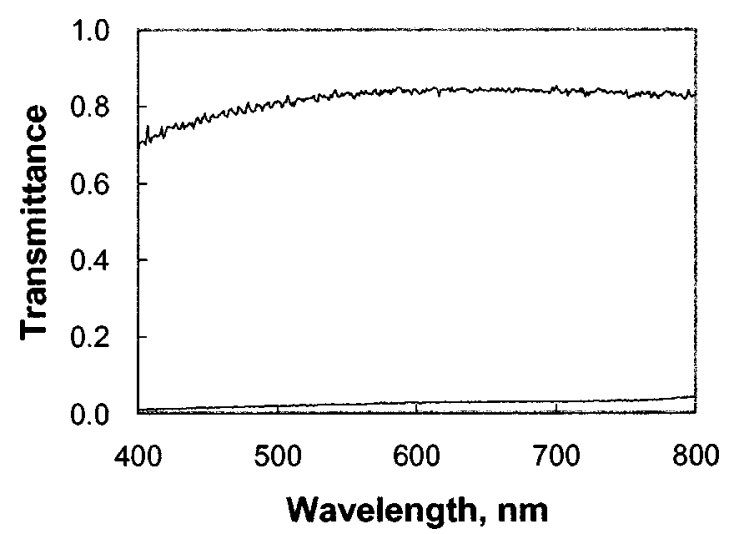

FIG. 3. The spectral bandwidth of a $10 \mu \mathrm{m}$ LC gel. Upper trace: $V$ $=10 \mathrm{~V}_{\text {rms }}$ and lower trace: $V=0$. A calcite polarizer was used to polarize the incident light.

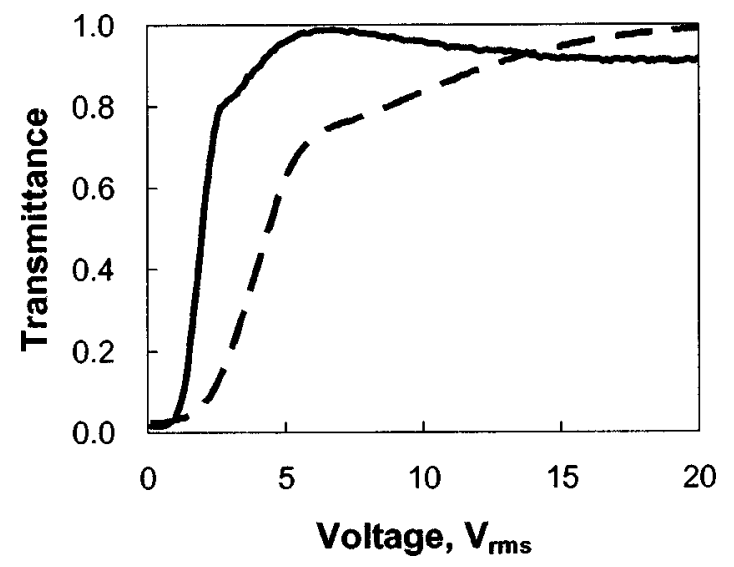

FIG. 4. Voltage-dependent normalized transmittance of the LC gels with monomer concentration of $0.4 \mathrm{wt} \%$ at 30 (solid lines) and 100 (dashed lines) $\mu \mathrm{W} / \mathrm{cm}^{2} \mathrm{UV}$ intensities.

the device functions like crossed polarizers. An extinction ratio greater than 150:1 was measured using two $c=0.4 \%$ LC gels and an unpolarized $\mathrm{He}-\mathrm{Ne}$ laser.

Figure 3 shows the measured transmission spectra of the $d=10 \mu \mathrm{m}$ LC gel in the voltage-off and -on states. The onstate voltage is $10 \mathrm{~V}_{\text {rms }}$. The spectral bandwidth covers the whole visible region. Such an anisotropic LC gel can be used as a white-light switchable polarizer. In the voltage-off state, the gel scatters the polarization along the buffing direction so that the transmitted light becomes linearly polarized. In the voltage-on state, the gel is highly transparent and is independent of polarization.

We also studied the UV intensity effect on the gel formation. Figure 4 compares the voltage-dependent transmittance of the LC gels with same monomer concentration ( $c$ $=0.4 \%$ ) but prepared at two different UV intensities: 30 and

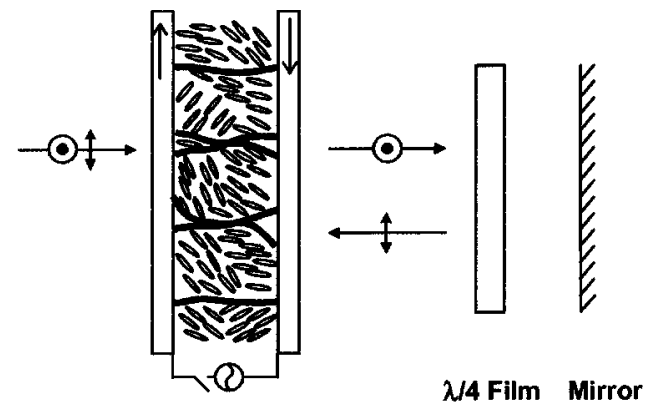

(a) Voltage-Off

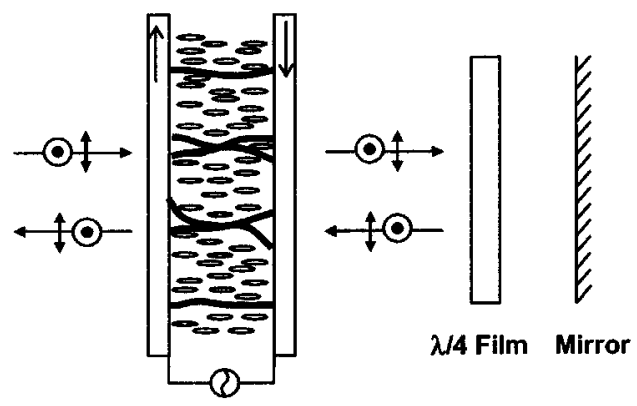

(b) Voltage-On

FIG. 5. A reflective display consists of a normal-mode LC gel, a quarterwave film, and a reflector: (a) voltage-off state and (b) voltage-on state. The arrows in the substrates indicate the rubbing directions. 
$100 \mu \mathrm{w} / \mathrm{cm}^{2}$. For the weaker UV exposure, the polymerization process is slower leading to a coarser polymer network and lower operating voltage. Under such a circumstance, the polymerization process is more complete and the polymer network is more stable resulting in a better dark state.

The rise and fall time of the $c=0.4 \%$ and $d=10 \mu \mathrm{m} \mathrm{LC}$ gel was measured to be 1.5 and $192 \mathrm{~ms}$, respectively. To reduce the fall time, we could increase the monomer concentration or decrease the cell gap. Increasing monomer concentration would lead to a stronger anchoring force between the polymer network and LC interfaces. Therefore, the threshold voltage is increased but the decay time is decreased. On the other hand, decreasing the cell gap would substantially reduce the decay time except that the contrast ratio is also decreased. To boost the contrast ratio, a high birefringence LC mixture should be employed.

We have applied the LC gel for reflective display. Figure 5 shows the device configuration which consists of an anisotropic gel cell, a broadband quarter-wave film, and a reflector. The angle between the optical axis of the wave plate and cell rubbing direction is $45^{\circ}$. In a voltage-off state, the input polarization ( $p$ wave) which is parallel to the cell rubbing direction is highly scattered at the first pass. The transmitted $s$ wave traverses the $\lambda / 4$ film twice and is converted to $p$ wave. During the return path, this $p$ wave is again scattered resulting in a translucent state. In the voltage-on state, the cell does not affect the incident polarization at all. The incident light traverses through the cell and wave plate and is reflected back by the reflector with high efficiency. The contrast ratio (CR) of the $c=0.4 \%$ and $d=10 \mu \mathrm{m} \mathrm{LC}$ gel was measured to be $\sim 60: 1$. For the $c=0.6 \%$ and $d=8 \mu \mathrm{m} \mathrm{LC}$ gel, we obtained a CR of $\sim 40: 1$, rise time; $\sim 2 \mathrm{~ms}$, and fall time; $\sim 65 \mathrm{~ms}$. To reduce response time further while retaining a good contrast ratio, a high birefringence LC mixture could be used and cell gap reduced.

In conclusion, a normal-mode LC gel is demonstrated. Such device exhibits a modest contrast ratio and low operating voltage. The potential applications of LC gel can be found in switchable polarizers, telecom optical switches, and reflective displays.

This work was supported by AFOSR under Contract No. F49620-01-1-0377.

${ }^{1}$ R. A. M. Hikmet and H. J. Boots, Phys. Rev. E 51, 5824 (1995).

${ }^{2}$ R. A. M. Hikmet, Liq. Cryst. 9, 405 (1991).

${ }^{3}$ H. Ren and S. T. Wu, Appl. Phys. Lett. 81, 1432 (2002).

${ }^{4}$ S. T. Wu and D. K. Yang, Reflective Liquid-Crystal Displays (Wiley, Chichester, 2001), Chap. 7.

${ }^{5}$ D. K. Yang, L. C. Chien, and Y. K. Fung, in Liquid Crystals in Complex Geometries Formed by Polymer and Porous Networks, edited by G. P. Crawford and S. Zumer (Taylor \& Francis, London, 1996), Chap. 5. 